\title{
Congruences for overpartitions with restricted odd differences
}

\author{
M. S. Mahadeva Naika ${ }^{1}$ D. S. Gireesh ${ }^{1,2}$
}

Received: 8 June 2017 / Accepted: 23 August 2018 / Published online: 30 August 2018

(c) African Mathematical Union and Springer-Verlag GmbH Deutschland, ein Teil von Springer Nature 2018

\begin{abstract}
In recent work, Bringmann et al. used $q$-difference equations to compute a two-variable $q$ hypergeometric generating function for the number of overpartitions where (i) the difference between two successive parts may be odd only if the larger of the two is overlined, and (ii) if the smallest part is odd then it is overlined, given by $\bar{t}(n)$. They also established the twovariable generating function for the same overpartitions where (i) consecutive parts differ by a multiple of $(k+1)$ unless the larger of the two is overlined, and (ii) the smallest part is overlined unless it is divisible by $k+1$, enumerated by $\bar{t}^{(k)}(n)$. As an application they proved that $\bar{t}(n) \equiv 0(\bmod 3)$ if $n$ is not a square. In this paper, we extend the study of congruence properties of $\bar{t}(n)$, and we prove congruences modulo 3 and 6 for $\bar{t}(n)$, congruences modulo 2 and 4 for $\bar{t}^{(3)}(n)$ and $\bar{t}^{(7)}(n)$, congruences modulo 4 and 5 for $\bar{t}^{(4)}(n)$, and congruences modulo 3,6 and 12 for $\bar{t}^{(8)}(n)$.
\end{abstract}

Keywords Partitions · Overpartitions · Congruences

Mathematics Subject Classification 05A17 · 11P82

\section{Introduction}

For $|a b|<1$, Ramanujan's general theta function $f(a, b)$ is defined by

$$
f(a, b):=\sum_{n=-\infty}^{\infty} a^{n(n+1) / 2} b^{n(n-1) / 2} .
$$

$凶$ D. S. Gireesh

gireeshdap@gmail.com

M. S. Mahadeva Naika

msmnaika@rediffmail.com

1 Department of Mathematics, Bangalore University, Central College Campus, Bengaluru, Karnataka 560 001, India

2 Department of Mathematics, M. S. Ramaiah University of Applied Sciences, Peenya, Bengaluru, Karnataka 560 058, India 
Using Jacobi's famous triple product identity [5, Entry 19, p.35], (1.1) takes the form

$$
f(a, b)=(-a ; a b)_{\infty}(-b ; a b)_{\infty}(a b ; a b)_{\infty},
$$

where $(a ; b)_{\infty}=(1-a)(1-a b)\left(1-a b^{2}\right) \cdots$.

Throughout this paper, we will use

$$
f_{k}:=\left(q^{k} ; q^{k}\right)_{\infty}
$$

The most important special cases of $f(a, b)$ are

$$
\begin{aligned}
& \varphi(q):=f(q, q)=1+2 \sum_{n \geq 1} q^{n^{2}}=\left(-q ; q^{2}\right)_{\infty}^{2}\left(q^{2} ; q^{2}\right)_{\infty}=\frac{f_{2}^{5}}{f_{1}^{2} f_{4}^{2}}, \\
& \psi(q):=f\left(q, q^{3}\right)=\sum_{n \geq 0} q^{n(n+1) / 2}=\frac{\left(q^{2} ; q^{2}\right)_{\infty}}{\left(q ; q^{2}\right)_{\infty}}=\frac{f_{2}^{2}}{f_{1}}
\end{aligned}
$$

and

$$
f(-q):=f\left(-q,-q^{2}\right)=\sum_{n=-\infty}^{\infty}(-1)^{n} q^{n(3 n-1) / 2}=(q ; q)_{\infty}=f_{1} .
$$

Equation (1.4) is the famous pentagonal number theorem [2, pp. 9-12].

A partition of a positive integer $n$ is a non-increasing sequence of positive integers whose sum is $n$; the number of partitions of $n$ is denoted by $p(n)$. It is well known that the generating function of $p(n)$ is

$$
\sum_{n \geq 0} p(n) q^{n}=\frac{1}{(q ; q)_{\infty}}
$$

Ramanujan's [14], [15, pp. 210-213], three famous congruences satisfied by $p(n)$ are

$$
\begin{aligned}
p(5 n+4) \equiv 0 & (\bmod 5), \\
p(7 n+5) \equiv 0 & (\bmod 7), \\
p(11 n+6) \equiv 0 & (\bmod 11) .
\end{aligned}
$$

Motivated by these congruences mathematicians are engaged in finding such congruences for different partition functions. One of the partition functions we discuss here is overpartitions. An overpartition of $n$ is a partition of $n$ in which the first occurrence (equivalently, the final occurrence) of a part may be overlined. Let $\bar{p}(n)$ denote the number of overpartitions of $n$. Corteel and Lovejoy [9] showed that the generating function of $\bar{p}(n)$ is

$$
\sum_{n \geq 0} \bar{p}(n) q^{n}=\frac{(-q ; q)_{\infty}}{(q ; q)_{\infty}}=\frac{1}{\varphi(-q)} .
$$

For example, the 24 overpartitions of 5 are

$$
\begin{aligned}
& 5, \overline{5}, 4+1, \overline{4}+1,4+\overline{1}, \overline{4}+\overline{1}, 3+2, \overline{3}+2,3+\overline{2}, \overline{3}+\overline{2}, 3+1+1, \overline{3}+1+1, \\
& 3+1+\overline{1}, \overline{3}+1+\overline{1}, 2+2+1, \overline{2}+2+1,2+2+\overline{1}, 2+\overline{2}+\overline{1}, 2+1+1+1, \\
& \overline{2}+1+1+1,2+1+1+\overline{1}, \overline{2}+1+1+\overline{1}, 1+1+1+1+1,1+1+1+1+\overline{1} .
\end{aligned}
$$

Andrews [3] defined combinatorial objects that he called singular overpartitions which are overpartitions in which no part is divisible by $\delta$ and only parts $\equiv \pm i(\bmod \delta)$ may be 
overlined. The number of singular overpartitions of $n$ is denoted by $\bar{C}_{\delta, i}(n)$. The ten singular overpartitions counted $\bar{C}_{3,1}(4)$ are

$4, \overline{4}, 2+2, \overline{2}+2,2+1+1, \overline{2}+1+1,2+\overline{1}+1, \overline{2}+\overline{1}+1,1+1+1+1, \overline{1}+1+1+1$.

For $\delta \geq 3$ and $1<i<\left\lfloor\frac{\delta}{2}\right\rfloor$, the generating function for $\bar{C}_{\delta, i}(n)$ is

$$
\sum_{n \geq 0} \bar{C}_{\delta, i}(n) q^{n}=\frac{\left(q^{\delta} ; q^{\delta}\right)_{\infty}\left(-q^{i} ; q^{\delta}\right)_{\infty}\left(-q^{\delta-i} ; q^{\delta}\right)_{\infty}}{(q ; q)_{\infty}}
$$

Andrews found that for each $n \geq 0$,

$$
\bar{C}_{3,1}(9 n+3) \equiv \bar{C}_{3,1}(9 n+6) \equiv 0(\bmod 3),
$$

and also that, for all $n \geq 0, \bar{C}_{3,1}(n)=\bar{A}_{3}(n)$, where $\bar{A}_{3}(n)$ is the number of overpartitions of $n$ into parts not divisible by 3 . To know more about overpartitions one can see $[1,7,13]$.

Let $\bar{t}(n)$ denote the number of overpartitions of $n$ where (i) the difference between two successive parts may be odd only if the larger of two is overlined, and (ii) if the smallest part is odd then it is overlined. Let $\bar{s}(n)$ denote the number of overpartitions counted by $\bar{t}(n)$ but with odd smallest part. Let $\bar{t}(m, n)$ and $\bar{s}(m, n)$ denote the number of overpartitions counted by $\bar{t}(n)($ resp. $\bar{s}(n))$ having $m$ parts. For example, the nine overpartitions counted by $\bar{t}(5)$ are

$\overline{5}, \overline{4}+\overline{1}, \overline{3}+2, \overline{3}+\overline{2}, 3+1+\overline{1}, \overline{3}+1+\overline{1}, 2+\overline{2}+\overline{1}, \overline{2}+1+1+\overline{1}, 1+1+1+1+\overline{1}$ and seven overpartitions counted by $\bar{s}(5)$ are

$$
\overline{5}, \overline{4}+\overline{1}, 3+1+\overline{1}, \overline{3}+1+\overline{1}, 2+\overline{2}+\overline{1}, \overline{2}+1+1+\overline{1}, 1+1+1+1+\overline{1} .
$$

Bringmann et al. [6] have proved the following identities:

$$
\begin{aligned}
\sum_{m, n \geq 0} \bar{t}(m, n) x^{m} q^{n} & =\frac{(-x q ; q)_{\infty}}{(x q ; q)_{\infty}}\left(1+\sum_{n \geq 1} \frac{\left(-q^{3} ; q^{3}\right)_{n-1}(-x)^{n} q^{n}}{(-q ; q)_{n-1}\left(q^{2} ; q^{2}\right)_{n}}\right) \\
\sum_{m, n \geq 1} \bar{s}(m, n) x^{m} q^{n} & =\sum_{n \geq 1} \frac{\left(q^{3} ; q^{3}\right)_{n-1} x^{n} q^{n}}{(q ; q)_{n-1}\left(q^{2} ; q^{2}\right)_{n}}
\end{aligned}
$$

For some particular values of $x$ and using the mock theta functions $\bar{\gamma}(q)$ and $\bar{\chi}(q)$ defined by

$$
\bar{\gamma}(q):=\sum_{n \geq 0} \frac{(-1 ; q)_{n}(q ; q)_{n} q^{\left(\begin{array}{c}
n+1 \\
2
\end{array}\right)}}{\left(q^{3} ; q^{3}\right)_{n}}
$$

and

$$
\bar{\chi}(q):=\sum_{n \geq 0} \frac{(-1 ; q)_{n}(-q ; q)_{n} q^{\left(\begin{array}{c}
n+1 \\
2
\end{array}\right)}}{\left(-q^{3} ; q^{3}\right)_{n}}
$$


Bringmann et al. [6] have also proved the following identities:

$$
\begin{aligned}
\sum_{n \geq 0} \bar{t}(n) q^{n} & =\frac{\left(q^{3} ; q^{3}\right)_{\infty}}{(q ; q)_{\infty}\left(q^{2} ; q^{2}\right)_{\infty}}, \\
\sum_{n \geq 0}\left(\bar{t}_{+}(n)-\bar{t}_{-}(n)\right) q^{n} & =\frac{\left(-q^{3} ; q^{3}\right)_{\infty}}{(-q ; q)_{\infty}^{3}} \bar{\chi}(q), \\
\sum_{n=1}^{\infty} \overline{\bar{s}}(n) q^{n} & :=1+3 \sum_{n \geq 1}\left(\bar{s}_{+}(n)-\bar{s}_{-}(n)\right) q^{n} \\
& =\frac{\left(-q^{3} ; q^{3}\right)_{\infty}}{(-q ; q)_{\infty}^{3}}, \\
1+3 \sum_{n \geq 1} \bar{s}(n) q^{n} & =\frac{\left(q^{3} ; q^{3}\right)_{\infty}}{(q ; q)_{\infty}\left(q^{2} ; q^{2}\right)_{\infty}} \bar{\chi}(q),
\end{aligned}
$$

where $\bar{t}_{+}(n)$ (resp. $\left.\bar{s}_{+}(n)\right)$ denotes the number of overpartitions counted by $\bar{t}(n)(\operatorname{resp} . \bar{s}(n))$ with largest part even and $\bar{t}_{-}(n)$ (resp. $\left.\bar{s}_{-}(n)\right)$ denotes the number of overpartitions counted by $\bar{t}(n)($ resp. $\bar{s}(n))$ with largest part odd. Bringmann et al. [6] have also proved the following congruence identities:

For a prime $\ell \neq 2,3$, and $n \geq 0$,

$$
\begin{aligned}
\bar{t}_{+}\left(\ell^{2} n\right)+ & \left(\left(\frac{-n}{\ell}\right)-\ell-1\right) \bar{t}_{+}(n)+\ell \bar{t}_{+}\left(\frac{n}{\ell^{2}}\right) \\
& \equiv \bar{t}_{-}\left(\ell^{2} n\right)+\left(\left(\frac{-n}{\ell}\right)-\ell-1\right) \bar{t}_{-}(n)+\ell \bar{t}_{-}\left(\frac{n}{\ell^{2}}\right) \quad(\bmod 3) .
\end{aligned}
$$

For $n \geq 1$,

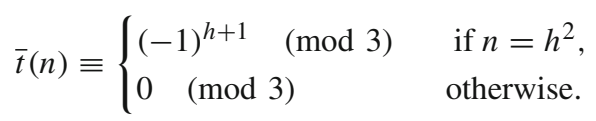

Bringmann et al. [6] generalized (1.12) as

$$
\sum_{n \geq 0} \bar{t}^{(k)}(n) q^{n}=\frac{\left(q^{k+1} ; q^{k+1}\right)_{\infty}}{\left(q^{k} ; q^{k}\right)_{\infty}(q ; q)_{\infty}}
$$

where $\bar{t}^{(k)}(n)$ denotes the number of overpartitions of $n$ where (i) consecutive parts differ by a multiple of $(k+1)$ unless the larger of the two is overlined, and (ii) the smallest part is overlined unless it is divisible by $k+1$. $\overline{\bar{s}}(n)$.

Chern et al. [8] studied Ramanujan-type congruences for the partition functions $\bar{t}(n)$ and

By (1.11) and (1.15), we deduce that

$$
1+3 \sum_{n \geq 1} \bar{s}(n) q^{n}=\frac{\left(q^{3} ; q^{3}\right)_{\infty}}{(q ; q)_{\infty}\left(q^{2} ; q^{2}\right)_{\infty}}\left(1+\sum_{n \geq 1} \frac{(-1 ; q)_{n}(-q ; q)_{n} q^{\left(\begin{array}{c}
n+1 \\
2
\end{array}\right)}}{\left(-q^{3} ; q^{3}\right)_{n}}\right)
$$

which implies that

$$
1+\sum_{n \geq 1} \bar{s}(n) q^{n} \equiv \frac{\left(q^{3} ; q^{3}\right)_{\infty}}{(q ; q)_{\infty}\left(q^{2} ; q^{2}\right)_{\infty}} \quad(\bmod 2)
$$


Applying (1.12) in (1.20), we find that

$$
\sum_{n \geq 0} \bar{s}(n) q^{n} \equiv \sum_{n \geq 0} \bar{t}(n) q^{n} \quad(\bmod 2)
$$

from which we can say that "For any nonnegative integer $n, \bar{t}(n)$ is even (or odd) iff $\bar{s}(n)$ is even (or odd)".

Motivated by the above work, in this paper, we extend the study of congruence properties of overpartitions with restricted odd differences. In Sect. 3, we prove congruences modulo 6 for $\bar{t}(n)$, while in Sects. 4-7, we prove congruences modulo 2, 4 for $\bar{t}^{(3)}(n)$ and $\bar{t}^{(7)}(n)$, congruences modulo 4 and 5 for $\bar{t}^{(4)}(n)$ and congruences modulo 3,6 , and 12 for $\bar{t}^{(8)}(n)$.

\section{Preliminaries}

In this section, we recall 2-dissection identities for certain quotients of theta functions and $p$-dissection identities for theta functions $f(-q)$ and $\psi(q)$ which plays a key role in proving our main results.

Lemma 2.1 The following 2-dissections hold.

$$
\begin{aligned}
\frac{1}{f_{1}^{2}} & =\frac{f_{8}^{5}}{f_{2}^{5} f_{16}^{2}}+2 q \frac{f_{4}^{2} f_{16}^{2}}{f_{2}^{5} f_{8}}, \\
f_{1}^{4} & =\frac{f_{4}^{10}}{f_{2}^{2} f_{8}^{4}}-4 q \frac{f_{2}^{2} f_{8}^{4}}{f_{4}^{2}}, \\
\frac{1}{f_{1}^{4}} & =\frac{f_{4}^{14}}{f_{2}^{14} f_{8}^{4}}+4 q \frac{f_{4}^{2} f_{8}^{4}}{f_{2}^{10}}, \\
\frac{f_{3}}{f_{1}} & =\frac{f_{4} f_{6} f_{16} f_{24}^{2}}{f_{2}^{2} f_{8} f_{12} f_{48}}+q \frac{f_{6} f_{8}^{2} f_{48}}{f_{2}^{2} f_{16} f_{24}}, \\
\frac{1}{f_{1} f_{3}} & =\frac{f_{8}^{2} f_{12}^{5}}{f_{2}^{2} f_{4} f_{6}^{4} f_{24}^{2}}+q \frac{f_{4}^{5} f_{24}^{2}}{f_{2}^{4} f_{6}^{2} f_{8}^{2} f_{12}}, \\
\frac{f_{1}^{3}}{f_{3}} & =\frac{f_{4}^{3}}{f_{12}}-3 q \frac{f_{2}^{2} f_{12}^{3}}{f_{4} f_{6}^{2}}, \\
\frac{f_{3}}{f_{1}^{3}} & =\frac{f_{4}^{6} f_{6}^{3}}{f_{2}^{9} f_{12}^{2}}+3 q \frac{f_{4}^{2} f_{6} f_{12}^{2}}{f_{2}^{7}}, \\
\frac{f_{9}}{f_{1}} & =\frac{f_{12}^{3} f_{18}}{f_{2}^{2} f_{6} f_{36}}+q \frac{f_{4}^{2} f_{6} f_{36}}{f_{2}^{3} f_{12}}, \\
\frac{f_{5}}{f_{1}} & =\frac{f_{8} f_{20}^{2}}{f_{2}^{2} f_{40}}+q \frac{f_{4}^{3} f_{10} f_{40}}{f_{2}^{3} f_{8} f_{20}}, \\
\frac{1}{f_{1} f_{7}} & =\frac{f_{16}^{2} f_{56}^{5}}{f_{2}^{2} f_{8} f_{14}^{2} f_{28}^{2} f_{112}^{2}}+q \frac{f_{4}^{2} f_{28}^{2}}{f_{2}^{3} f_{14}^{3}}+q^{6} \frac{f_{8}^{5} f_{112}^{2}}{f_{2}^{2} f_{4}^{2} f_{14}^{2} f_{16}^{2} f_{56}}, \\
f_{1} f_{7} & =\frac{f_{2} f_{14} f_{16}^{2} f_{56}^{5}}{f_{4} f_{8} f_{28}^{3} f_{112}^{2}}-q f_{4} f_{28}+q^{6} \frac{f_{2} f_{8}^{5} f_{14} f_{112}^{2}}{f_{4}^{3} f_{16}^{2} f_{28} f_{56}} . \\
& =1
\end{aligned}
$$


Equations (2.1)-(2.3) are consequences of dissection formulas of Ramanujan, collected in Berndt's book [5, Entry 25 p. 40]. Xia and Yao [17] proved (2.4) by employing Jacobi triple product identity. Equation (2.5) was proved by Baruah and Ojah [4] and (2.6) was proved by Hirschhorn, Garvan, and Borwein [11]. Replacing $q$ by $-q$ in (2.6) and using the relation

$$
(-q ;-q)_{\infty}=\frac{f_{2}^{3}}{f_{1} f_{4}}
$$

we obtain (2.7). Equation (2.8) was proved by Xia and Yao [18]. (2.9) was proved by Hirschhorn and Sellers [12]. (2.10) and (2.11) was proved by Xia [16, Lemma 3.4]

Lemma 2.2 [10, Theorem 2.1] For any odd prime $p$,

$$
\psi(q)=\sum_{m=0}^{\frac{p-3}{2}} q^{\frac{m^{2}+m}{2}} f\left(q^{\frac{p^{2}+(2 m+1) p}{2}}, q^{\frac{p^{2}-(2 m+1) p}{2}}\right)+q^{\frac{p^{2}-1}{8}} \psi\left(q^{p^{2}}\right) .
$$

Furthermore, $\frac{m^{2}+m}{2} \not \equiv \frac{p^{2}-1}{8}(\bmod p)$ for $0 \leq m \leq \frac{p-3}{2}$.

Lemma 2.3 [10, Theorem 2.2] For any prime $p \geq 5$,

$$
\begin{aligned}
f_{1}= & \sum_{\substack{k=-\frac{p-1}{2} \\
k \neq( \pm p-1) / 6}}^{\frac{p-1}{2}}(-1)^{k} q^{\frac{3 k^{2}+k}{2}} f\left(-q^{\frac{3 p^{2}+(6 k+1) p}{2}},-q^{\frac{3 p^{2}-(6 k+1) p}{2}}\right) \\
& +(-1)^{\frac{ \pm p-1}{6}} q^{\frac{p^{2}-1}{24}} f_{p^{2}} .
\end{aligned}
$$

Furthermore, for $-(p-1) / 2 \leq k \leq(p-1) / 2$ and $k \neq \frac{ \pm p-1}{6}$,

$$
\frac{3 k^{2}+k}{2} \not \equiv \frac{p^{2}-1}{24}(\bmod p),
$$

where $\frac{ \pm p-1}{6}:= \begin{cases}\frac{p-1}{6}, & p \equiv 1 \bmod 6 \\ \frac{-p-1}{6}, & p \equiv-1 \bmod 6 .\end{cases}$

\section{Congruences for $\bar{t}(n)$}

In this section, we prove infinite family of congruences modulo 6 for $\bar{t}(n)$ by using dissections of theta function identities.

Theorem 3.1 If $p$ is any prime $p \geq 5$ such that $\left(\frac{-2}{p}\right)=-1$ and $1 \leq j \leq p-1$, then for any nonnegative integer $\alpha$

$$
\sum_{n \geq 0} \bar{t}\left(8 p^{2 \alpha} n+3 p^{2 \alpha}\right) q^{n} \equiv 3 \psi(q) f_{6} \quad(\bmod 6)
$$

and for $n \geq 0$,

$$
\bar{t}\left(8 p^{2 \alpha+1}(p n+j)+3 p^{2 \alpha+2}\right) \equiv 0 \quad(\bmod 6) .
$$


Proof From (1.12) and (2.4), we find that

$$
\sum_{n \geq 0} \bar{t}(n) q^{n}=\frac{f_{4} f_{6} f_{16} f_{24}^{2}}{f_{2}^{3} f_{8} f_{12} f_{48}}+q \frac{f_{6} f_{8}^{2} f_{48}}{f_{2}^{3} f_{16} f_{24}}
$$

which yields

$$
\sum_{n \geq 0} \bar{t}(2 n+1) q^{n}=\frac{f_{3} f_{4}^{2} f_{24}}{f_{1}^{3} f_{8} f_{12}}
$$

Invoking (2.7) and (3.4), we see that

$$
\sum_{n \geq 0} \bar{t}(2 n+1) q^{n}=\frac{f_{4}^{8} f_{6}^{3} f_{24}}{f_{2}^{9} f_{8} f_{12}^{3}}+3 q \frac{f_{4}^{4} f_{6} f_{12} f_{24}}{f_{2}^{7} f_{8}} .
$$

Equating the coefficients of $q^{2 n+1}$, dividing throughout by $q$ and then replacing $q^{2}$ by $q$ in (3.5), we find that

$$
\sum_{n \geq 0} \bar{t}(4 n+3) q^{n}=3 \frac{f_{2}^{4} f_{3} f_{6} f_{12}}{f_{1}^{7} f_{4}}
$$

Substituting (2.3) and (2.7) into (3.6), we find that

$$
\sum_{n \geq 0} \bar{t}(4 n+3) q^{n}=3 \frac{f_{2}^{4} f_{6} f_{12}}{f_{4}}\left(\frac{f_{4}^{6} f_{6}^{3}}{f_{2}^{9} f_{12}^{2}}+3 q \frac{f_{4}^{2} f_{6} f_{12}^{2}}{f_{2}^{7}}\right)\left(\frac{f_{4}^{14}}{f_{2}^{14} f_{8}^{4}}+4 q \frac{f_{4}^{2} f_{8}^{4}}{f_{2}^{10}}\right),
$$

from which we get

$$
\sum_{n \geq 0} \bar{t}(8 n+3) q^{n}=3 \frac{f_{2}^{19} f_{3}^{4}}{f_{1}^{19} f_{4}^{4} f_{6}}+36 q \frac{f_{2}^{3} f_{3}^{2} f_{4}^{4} f_{6}^{3}}{f_{1}^{13}}
$$

By the binomial theorem, it is easy to see that for all positive integers $r$ and $m$

$$
\begin{aligned}
& f_{r}^{2 m} \equiv f_{2 r}^{m} \quad(\bmod 2), \\
& f_{r}^{4 m} \equiv f_{2 r}^{2 m} \quad(\bmod 4) .
\end{aligned}
$$

From (3.9), it follows that

$$
\frac{f_{2}^{19} f_{3}^{4}}{f_{1}^{19} f_{4}^{4} f_{6}} \equiv \frac{f_{2}^{2} f_{6}}{f_{1}}=\psi(q) f_{6} \quad(\bmod 2)
$$

In view of (3.11), we can rewrite (3.8) as

$$
\sum_{n \geq 0} \bar{t}(8 n+3) q^{n} \equiv 3 \psi(q) f_{6} \quad(\bmod 6) .
$$

From here our proof relies on mathematical induction. The congruence (3.12) is the case $\alpha=0$ of (3.1). Now assume that (3.1) holds for some $\alpha \geq 0$. Substituting (2.12) and (2.13) 
into (3.12), we deduce that

$$
\begin{aligned}
& \sum_{n \geq 0} \bar{t}\left(8 p^{2 \alpha} n+3 p^{2 \alpha}\right) q^{n} \\
& \equiv 3\left(\sum_{m=0}^{\frac{p-3}{2}} q^{\frac{m^{2}+m}{2}} f\left(q^{\frac{p^{2}+(2 m+1) p}{2}}, q^{\frac{p^{2}-(2 m+1) p}{2}}\right)+q^{\frac{p^{2}-1}{8}} \psi\left(q^{p^{2}}\right)\right) \\
& \quad \times\left(\sum_{\substack{k=-\frac{p-1}{2} \\
k \neq( \pm p-1) / 6}}^{\frac{p-1}{2}} q^{6 \times \frac{3 k^{2}+k}{2}} f\left(-q^{6 \times \frac{3 p^{2}+(6 k+1) p}{2}},-q^{\left.6 \times \frac{3 p^{2}-(6 k+1) p}{2}\right)}\right)\right. \\
& +q^{6 \times \frac{p^{2}-1}{24} f_{6 p^{2}}}(\bmod 6) .
\end{aligned}
$$

For a prime $p \geq 5,-(p-1) / 2 \leq k \leq(p-1) / 2$ and $0 \leq m \leq(p-3) / 2$, consider the congruence

$$
\frac{m^{2}+m}{2}+6 \times \frac{3 k^{2}+k}{2} \equiv \frac{9 p^{2}-9}{24}(\bmod p),
$$

which is equivalent to

$$
(2 m+1)^{2}+2(6 k+1)^{2} \equiv 0(\bmod p) .
$$

Since $\left(\frac{-2}{p}\right)=-1$, the only solution of the above congruence is $m=(p-1) / 2$ and $k=( \pm p-1) / 6$. Therefore, extracting the terms containing $q^{p n+\frac{9 p^{2}-9}{24}}$ from both sides of (3.13), dividing throughout by $q^{\frac{9 p^{2}-9}{24}}$ and then replacing $q^{p}$ by $q$, we find that

$$
\sum_{n \geq 0} \bar{t}\left(8 p^{2 \alpha+1} n+3 p^{2 \alpha+2}\right) q^{n} \equiv 3 \psi\left(q^{p}\right) f_{6 p} \quad(\bmod 6),
$$

which yields

$$
\sum_{n \geq 0} \bar{t}\left(8 p^{2 \alpha+2} n+3 p^{2 \alpha+2}\right) q^{n} \equiv 3 \psi(q) f_{6} \quad(\bmod 6),
$$

which is the (3.1) with $\alpha+1$ for $\alpha$. Comparing the coefficients of $q^{p n+j}$, for $1 \leq j \leq p-1$, from both sides of (3.14), we arrive at (3.2).

\section{Congruences for $\bar{t}^{(3)}(n)$}

In this section, we prove congruences and infinite family of congruences modulo 2 and 4 for $\bar{t}^{(3)}(n)$. 
Theorem 4.1 If $n$ and $\alpha$ are any nonnegative integers, then

$$
\begin{aligned}
& \bar{t}^{(3)}\left(4^{\alpha} n\right)+\bar{t}^{(3)}(n) \equiv 0 \quad(\bmod 2), \\
& \bar{t}^{(3)}\left(4^{\alpha}(4 n+2)\right) \equiv 0 \quad(\bmod 2), \\
& \bar{t}^{(3)}\left(4^{\alpha}(4 n+3)\right) \equiv 0 \quad(\bmod 2), \\
& \bar{t}^{(3)}\left(4^{\alpha}(8 n+5)\right) \equiv 0 \quad(\bmod 2) \text {, } \\
& \bar{t}^{(3)}(24 n+1) \equiv\left\{\begin{array}{lll}
1 & (\bmod 2) & \text { if } n \text { is a pentagonal number, } \\
0 & (\bmod 2) & \text { otherwise, }
\end{array}\right. \\
& \bar{t}^{(3)}(6 n+3) \equiv 0 \quad(\bmod 4), \\
& \bar{t}^{(3)}(6 n+5) \equiv 0 \quad(\bmod 4), \\
& \bar{t}^{(3)}(16 n+10) \equiv 0 \quad(\bmod 4) \text {, } \\
& \bar{t}^{(3)}(16 n+14) \equiv 0 \quad(\bmod 4) \text {, } \\
& \bar{t}^{(3)}(24 n+19) \equiv 0 \quad(\bmod 4) \text {. }
\end{aligned}
$$

Proof Setting $k=3$ in (1.18) and using (2.5), we find that

$$
\sum_{n \geq 0} \bar{t}^{(3)}(n) q^{n}=\frac{f_{4}}{f_{1} f_{3}}=f_{4}\left(\frac{f_{8}^{2} f_{12}^{5}}{f_{2}^{2} f_{4} f_{6}^{4} f_{24}^{2}}+q \frac{f_{4}^{5} f_{24}^{2}}{f_{2}^{4} f_{6}^{2} f_{8}^{2} f_{12}}\right) .
$$

Extracting the coefficients of even and odd powers of $q$ on both sides of the above equation, we obtain

$$
\begin{aligned}
\sum_{n \geq 0} \bar{t}^{(3)}(2 n) q^{n} & =\frac{f_{4}^{2} f_{6}^{5}}{f_{1}^{2} f_{3}^{4} f_{12}^{2}}, \\
\sum_{n \geq 0} \bar{t}^{(3)}(2 n+1) q^{n} & =\frac{f_{2}^{6} f_{12}^{2}}{f_{1}^{4} f_{3}^{2} f_{4}^{2} f_{6}} .
\end{aligned}
$$

Using (3.9), (4.12) can be rewritten

$$
\sum_{n \geq 0} \bar{t}^{(3)}(2 n) q^{n} \equiv \frac{f_{2}^{3}}{f_{6}} \quad(\bmod 2)
$$

which yields

$$
\begin{aligned}
\sum_{n \geq 0} \bar{t}^{(3)}(4 n) q^{n} & \equiv \frac{f_{1}^{3}}{f_{3}} \equiv \sum_{n \geq 0} \bar{t}^{(3)}(n) q^{n} \quad(\bmod 2), \\
\bar{t}^{(3)}(4 n+2) & \equiv 0 \quad(\bmod 2) .
\end{aligned}
$$

Equating the coefficients of $q^{n}$ in (4.15) and by mathematical induction, we arrive at (4.1). Using (4.16) in (4.1), we obtain (4.2).

In view of (3.9), we have

$$
\frac{f_{2}^{6} f_{12}^{2}}{f_{1}^{4} f_{3}^{2} f_{4}^{2} f_{6}} \equiv f_{12} \quad(\bmod 2)
$$


By (4.13) and (4.17), we find that

$$
\sum_{n \geq 0} \bar{t}^{(3)}(2 n+1) q^{n} \equiv f_{12} \quad(\bmod 2)
$$

from which we obtain

$$
\begin{array}{rlr}
\bar{t}^{(3)}(4 n+3) & \equiv 0 \quad(\bmod 2), \\
\bar{t}^{(3)}(8 n+5) & \equiv 0 \quad(\bmod 2), \\
\sum_{n \geq 0} \bar{t}^{(3)}(24 n+1) q^{n} & \equiv f_{1} \quad(\bmod 2) .
\end{array}
$$

Equations (4.3) and (4.4) follows from (4.1), (4.19) and (4.20).

The result (4.5) is obtained from (4.21) and (1.4).

Thanks to (3.10),

$$
\frac{f_{2}^{6} f_{12}^{2}}{f_{1}^{4} f_{3}^{2} f_{4}^{2} f_{6}} \equiv \frac{f_{6}^{3}}{f_{3}^{2}} \quad(\bmod 4) .
$$

In view of (4.13) and (4.22),

$$
\sum_{n \geq 0} \bar{t}^{(3)}(2 n+1) q^{n} \equiv \frac{f_{6}^{3}}{f_{3}^{2}} \quad(\bmod 4),
$$

which yields the desired results (4.6) and (4.7).

By (3.10), we have

$$
\frac{f_{4}^{2} f_{6}^{5}}{f_{1}^{2} f_{3}^{4} f_{12}^{2}} \equiv \frac{f_{4}^{2} f_{6}^{3}}{f_{1}^{2} f_{12}^{2}} \quad(\bmod 4) .
$$

Using (2.1) in the right hand side of (4.24) and then applying the resulting equation in (4.12), we deduce that

$$
\sum_{n \geq 0} \bar{t}^{(3)}(2 n) q^{n} \equiv \frac{f_{4}^{2} f_{6}^{3}}{f_{12}^{2}}\left(\frac{f_{8}^{5}}{f_{2}^{5} f_{16}^{2}}+2 q \frac{f_{4}^{2} f_{16}^{2}}{f_{2}^{5} f_{8}}\right) \quad(\bmod 4) .
$$

Extracting the terms involving odd powers of $q$ on both sides of (4.25), we obtain

$$
\begin{aligned}
\sum_{n \geq 0} \bar{t}^{(3)}(4 n+2) q^{n} & \equiv 2 \frac{f_{2}^{4} f_{3}^{3} f_{8}^{2}}{f_{1}^{5} f_{4} f_{6}^{2}} \quad(\bmod 4) \\
& \equiv 2 \frac{f_{8}^{2}}{f_{1} f_{3}} \quad(\bmod 4) .
\end{aligned}
$$

But in view of (3.9), we can rewrite (2.5) as

$$
\frac{1}{f_{1} f_{3}} \equiv \frac{f_{8}}{f_{12}}+q \frac{f_{24}}{f_{4}} \quad(\bmod 2) .
$$

Substituting (4.27) into (4.26), we find that

$$
\sum_{n \geq 0} \bar{t}^{(3)}(4 n+2) q^{n} \equiv 2 \frac{f_{8}^{3}}{f_{12}}+2 q \frac{f_{8}^{2} f_{24}}{f_{4}} \quad(\bmod 4) .
$$

Equating the coefficients of $q^{4 n+2}$ and $q^{4 n+3}$ on both sides of (4.28), we arrive at (4.8) and (4.9). 
Extracting the coefficients of $q^{3 n}$ on both sides of (4.23) and then replacing $q^{3}$ by $q$, we find that

$$
\sum_{n \geq 0} \bar{t}^{(3)}(6 n+1) q^{n} \equiv \frac{f_{2}^{3}}{f_{1}^{2}}(\bmod 4) .
$$

Applying (2.1) in (4.29), we obtain

$$
\sum_{n \geq 0} \bar{t}^{(3)}(6 n+1) q^{n} \equiv \frac{f_{8}^{5}}{f_{2}^{2} f_{16}^{2}}+2 q \frac{f_{4}^{2} f_{16}^{2}}{f_{2}^{2} f_{8}} \quad(\bmod 4)
$$

In view of (3.9) and (3.10), we can rewrite the above equations as

$$
\sum_{n \geq 0} \bar{t}^{(3)}(6 n+1) q^{n} \equiv \frac{f_{8}}{f_{2}^{2}}+2 q \frac{f_{8}^{2} f_{16}}{f_{4}} \quad(\bmod 4)
$$

which yields

$$
\sum_{n \geq 0} \bar{t}^{(3)}(12 n+7) q^{n} \equiv 2 \frac{f_{4}^{2} f_{8}}{f_{2}} \quad(\bmod 4)
$$

If we equate the coefficients of $q^{2 n+1}$ on both sides of the above equation, we obtain (4.10).

Theorem 4.2 Let $p \geq 5$ be any prime such that $\left(\frac{-3}{p}\right)=-1$ and $1 \leq j \leq p-1$. Then for any nonnegative integers $\alpha$,

$$
\sum_{n \geq 0} \bar{t}^{(3)}\left(24 p^{2 \alpha} n+7 p^{2 \alpha}\right) q^{n} \equiv 2 \psi(q) f_{4} \quad(\bmod 4)
$$

and for each $n \geq 0$,

$$
\bar{t}^{(3)}\left(24 p^{2 \alpha+1}(p n+j)+7 p^{2 \alpha+2}\right) \equiv 0 \quad(\bmod 4) .
$$

Proof We prove (4.33) by mathematical induction. Extracting the terms involving even powers of $q$ on both sides of (4.32), we find that

$$
\sum_{n \geq 0} \bar{t}^{(3)}(24 n+7) q^{n} \equiv 2 \frac{f_{2}^{2} f_{4}}{f_{1}}=2 \psi(q) f_{4} \quad(\bmod 4)
$$


which is the $\alpha=0$ case of (4.33). Now assume that (4.33) holds for some $\alpha \geq 0$. Substituting (2.12) and (2.13) into (4.33), we deduce that

$$
\begin{aligned}
& \sum_{n \geq 0} \bar{t}^{(3)}\left(24 p^{2 \alpha} n+7 p^{2 \alpha}\right) q^{n} \\
& \equiv 2\left(\sum_{m=0}^{\frac{p-3}{2}} q^{\frac{m^{2}+m}{2}} f\left(q^{\frac{p^{2}+(2 m+1) p}{2}}, q^{\frac{p^{2}-(2 m+1) p}{2}}\right)+q^{\frac{p^{2}-1}{8}} \psi\left(q^{p^{2}}\right)\right) \\
& \times\left(\sum_{\substack{k=-\frac{p-1}{2} \\
k \neq( \pm p-1) / 6}}^{\frac{p-1}{2}} q^{4 \times \frac{3 k^{2}+k}{2}} f\left(-q^{4 \times \frac{3 p^{2}+(6 k+1) p}{2}},-q^{4 \times \frac{3 p^{2}-(6 k+1) p}{2}}\right)\right. \\
& \left.+q^{4 \times \frac{p^{2}-1}{24}} f_{4 p^{2}}\right) \quad(\bmod 4) \text {. }
\end{aligned}
$$

For a prime $p \geq 5,-(p-1) / 2 \leq k \leq(p-1) / 2$ and $0 \leq m \leq(p-3) / 2$, consider the congruence

$$
4 \times \frac{3 k^{2}+k}{2}+\frac{m^{2}+m}{2} \equiv \frac{7 p^{2}-7}{24}(\bmod p),
$$

that is

$$
(12 k+2)^{2}+3(2 m+1)^{2} \equiv 0(\bmod p) .
$$

Since $\left(\frac{-3}{p}\right)=-1$, the only solution of the above congruence is $k=( \pm p-1) / 6$ and $m=(p-1) / 2$. Therefore, extracting the terms containing $q^{p n+\frac{7 p^{2}-7}{24}}$ from both sides of (4.36), dividing throughout by $q^{\frac{7 p^{2}-7}{24}}$ and then replacing $q^{p}$ by $q$, we find that

$$
\sum_{n \geq 0} \bar{t}\left(24 p^{2 \alpha+1} n+7 p^{2 \alpha+2}\right) q^{n} \equiv 2 \psi\left(q^{p}\right) f_{4 p} \quad(\bmod 4),
$$

which yields

$$
\sum_{n \geq 0} \bar{t}\left(24 p^{2 \alpha+2} n+7 p^{2 \alpha+2}\right) q^{n} \equiv 2 \psi(q) f_{4} \quad(\bmod 4)
$$

which is the (4.33) with $\alpha+1$ for $\alpha$. Comparing the coefficients of $q^{p n+j}$, for $1 \leq j \leq p-1$, from both sides of (4.37), we arrive at (4.34).

Theorem 4.3 Let $p \geq 5$ be any prime with $\left(\frac{-2}{p}\right)=-1$ and $1 \leq j \leq p-1$. Then for any nonnegative integers $\alpha$,

$$
\sum_{n \geq 0} \bar{t}^{(3)}\left(16 p^{2 \alpha} n+6 p^{2 \alpha}\right) q^{n} \equiv 2 \psi(q) f_{6} \quad(\bmod 4)
$$

and for each $n \geq 0$,

$$
\bar{t}^{(3)}\left(16 p^{2 \alpha+1}(p n+j)+6 p^{2 \alpha+2}\right) \equiv 0 \quad(\bmod 4) .
$$


Proof Equating the coefficients of $q^{4 n+1}$, dividing throughout by $q$ and then replacing $q^{4}$ by $q$ in (4.28), we obtain

$$
\sum_{n \geq 0} \bar{t}^{(3)}(16 n+6) q^{n} \equiv 2 \frac{f_{2}^{2} f_{6}}{f_{1}} \equiv 2 \psi(q) f_{6} \quad(\bmod 4) .
$$

Rest of the proof is similar to that of Eqs. (3.1) and (3.2) in the Theorem 3.1, so we omit the proof here.

\section{Congruences for $\bar{t}^{(4)}(n)$}

In this section, we obtain congruences modulo 4 and 5 for $\bar{t}^{(4)}(n)$.

Theorem 5.1 If $n$ is any nonnegative integer, then

$$
\begin{aligned}
\bar{t}^{(4)}(8 n+6) & \equiv 0 \quad(\bmod 4), \\
\bar{t}^{(4)}(16 n+10) & \equiv 0 \quad(\bmod 4), \\
\bar{t}^{(4)}(16 n+2) & \equiv\left\{\begin{array}{lll}
2 & (\bmod 4) & \text { if } n \text { is a triangular number, } \\
0 & (\bmod 4) & \text { otherwise. }
\end{array}\right.
\end{aligned}
$$

Proof Setting $k=4$ in (1.18) and then using (2.9), we obtain

$$
\sum_{n \geq 0} \bar{t}^{(4)}(n) q^{n}=\frac{f_{8} f_{20}^{2}}{f_{2}^{2} f_{4} f_{40}}+q \frac{f_{4}^{2} f_{10} f_{40}}{f_{2}^{3} f_{8} f_{20}},
$$

which yields

$$
\sum_{n \geq 0} \bar{t}^{(4)}(2 n) q^{n}=\frac{f_{4} f_{10}^{2}}{f_{1}^{2} f_{2} f_{20}} .
$$

Combining (2.1) and (5.5), we find that

$$
\sum_{n \geq 0} \bar{t}^{(4)}(2 n) q^{n}=\frac{f_{4} f_{10}^{2}}{f_{2} f_{20}}\left(\frac{f_{8}^{5}}{f_{2}^{5} f_{16}^{2}}+2 q \frac{f_{4}^{2} f_{16}^{2}}{f_{2}^{5} f_{8}}\right) .
$$

Extracting the coefficients of odd powers of $q$ on both sides of the above equation, we obtain

$$
\sum_{n \geq 0} \bar{t}^{(4)}(4 n+2) q^{n}=2 \frac{f_{2}^{3} f_{5}^{2} f_{8}^{2}}{f_{1}^{6} f_{4} f_{10}} .
$$

It follows from (3.9) that

$$
\frac{f_{2}^{3} f_{5}^{2} f_{8}^{2}}{f_{1}^{6} f_{4} f_{10}} \equiv f_{4} f_{8} \equiv \frac{f_{8}^{2}}{f_{4}} \equiv \psi\left(q^{4}\right) \quad(\bmod 2) .
$$

Using (5.8), (5.7) can be reduced to

$$
\sum_{n \geq 0} \bar{t}^{(4)}(4 n+2) q^{n} \equiv 2 f_{4} f_{8} \equiv 2 \psi\left(q^{4}\right) \quad(\bmod 4) .
$$

Equating the coefficients of $q^{2 n+1}$ and $q^{4 n+2}$ on both sides of the above equation, we arrive at (5.1) and (5.2). 
Extracting the terms of (5.9) in which powers of $q$ is congruent to 0 modulo 4 , we obtain

$$
\sum_{n \geq 0} \bar{t}^{(4)}(16 n+2) q^{n} \equiv 2 \psi(q) \quad(\bmod 4)
$$

A positive integer $x$ is said to be triangular number, if it is of the form $\frac{x(x+1)}{2}$. The result (5.3) follows from (1.3) and (5.10).

Theorem 5.2 For any prime $p \geq 5$ with $\left(\frac{-2}{p}\right)=-1,1 \leq j \leq p-1$, and $\alpha \geq 0$, we have

$$
\sum_{n \geq 0} \bar{t}^{(4)}\left(16 p^{2 \alpha} n+2 p^{2 \alpha}\right) q^{n} \equiv 2 f_{1} f_{2} \quad(\bmod 4)
$$

and for each $n \geq 0$,

$$
\bar{t}^{(4)}\left(16 p^{2 \alpha+1}(p n+j)+2 p^{2 \alpha+2}\right) \equiv 0 \quad(\bmod 4) .
$$

Proof From (5.9), we have

$$
\sum_{n \geq 0} \bar{t}^{(4)}(16 n+2) q^{n} \equiv 2 f_{1} f_{2} \quad(\bmod 4)
$$

For a prime $p \geq 5$ and $-(p-1) / 2 \leq k, m \leq(p-1) / 2$, consider

$$
\frac{3 k^{2}+k}{2}+2 \times \frac{3 m^{2}+m}{2} \equiv \frac{3 p^{2}-3}{24}(\bmod p),
$$

which implies that

$$
(6 k+1)^{2}+2(6 m+1)^{2} \equiv 0(\bmod p) .
$$

Since $\left(\frac{-2}{p}\right)=-1$, the only solution of the above congruence is $k=m=( \pm p-1) / 6$. Therefore, using Lemma 2.3, we have

$$
\sum_{n \geq 0} \bar{t}^{(4)}\left(16\left(p^{2} n+\frac{3 p^{2}-3}{24}\right)+2\right) q^{n} \equiv 2 f_{1} f_{2} \quad(\bmod 4)
$$

Invoking (5.13) and (5.14), we arrive at

$$
\sum_{n \geq 0} \bar{t}^{(4)}\left(16 p^{2} n+2 p^{2}\right) q^{n} \equiv \sum_{n \geq 0} \bar{t}^{(4)}(16 n+2) q^{n} \equiv 2 f_{1} f_{2} \quad(\bmod 4) .
$$

The result (5.11) follows from the above equation and by induction on $\alpha$. 
Substituting (2.13) into (5.11), we deduce that

$$
\begin{aligned}
& \sum_{n \geq 0} \bar{t}^{(4)}\left(16 p^{2 \alpha} n+2 p^{2 \alpha}\right) q^{n} \\
& \equiv 2\left(\sum_{\substack{k=-\frac{p-1}{2} \\
k \neq( \pm p-1) / 6}}^{\frac{p-1}{2}} q^{\frac{3 k^{2}+k}{2}} f\left(-q^{\frac{3 p^{2}+(6 k+1) p}{2}},-q^{\frac{3 p^{2}-(6 k+1) p}{2}}\right)+q^{\frac{p^{2}-1}{24}} f_{p^{2}}\right) \\
& \quad \times\left(\sum_{\substack{k=-\frac{p-1}{2} \\
k \neq( \pm p-1) / 6}}^{\frac{p-1}{2}} q^{2 \times \frac{3 k^{2}+k}{2}} f\left(-q^{2 \times \frac{3 p^{2}+(6 k+1) p}{2}},-q^{2 \times \frac{3 p^{2}-(6 k+1) p}{2}}\right)\right. \\
& +q^{2 \times \frac{p^{2}-1}{24} f_{2 p^{2}}}(\bmod 4),
\end{aligned}
$$

which yields

$$
\sum_{n \geq 0} \bar{t}^{(4)}\left(16 p^{2 \alpha+1} n+2 p^{2 \alpha+2}\right) q^{n} \equiv f_{p} f_{2 p} \quad(\bmod 4) .
$$

Equating the coefficients of $q^{p n+j}$ for $j=1,2, \ldots, p-1$ in (5.17), we obtain (5.12).

Theorem 5.3 For $n \geq 0$, we have

$$
\bar{t}^{(4)}(16 n+6) \equiv 2 \triangle_{3}(n) \quad(\bmod 5)
$$

and

$$
\bar{t}^{(4)}(16 n+14) \equiv 0 \quad(\bmod 5)
$$

Proof Setting $k=4$ in (1.18), we obtain

$$
\sum_{n \geq 0} \bar{t}^{(4)}(n) q^{n}=\frac{f_{5}}{f_{1} f_{4}} .
$$

In view of (3.10), we can rewrite (5.20) as

$$
\sum_{n \geq 0} \bar{t}^{(4)}(n) q^{n} \equiv \frac{f_{1}^{4}}{f_{4}} \quad(\bmod 5)
$$

Substituting (2.2) into (5.21) and then extracting even powers of $q$ on both sides, we obtain

$$
\begin{aligned}
\sum_{n \geq 0} \bar{t}^{(4)}(2 n) q^{n} & \equiv \frac{f_{2}^{9}}{f_{1}^{2} f_{4}^{4}} \quad(\bmod 5) \\
& \equiv \frac{f_{2}^{9}}{f_{4}^{4}}\left(\frac{f_{8}^{5}}{f_{2}^{5} f_{16}^{2}}+2 q \frac{f_{4}^{2} f_{16}^{2}}{f_{2}^{5} f_{8}}\right) \quad(\bmod 5)
\end{aligned}
$$


which implies that

$$
\begin{aligned}
\sum_{n \geq 0} \bar{t}^{(4)}(4 n+2) q^{n} & \equiv 2 \frac{f_{1}^{4} f_{8}^{2}}{f_{2}^{2} f_{4}} \quad(\bmod 5) \\
& \equiv 2 \frac{f_{8}^{2}}{f_{2}^{2} f_{4}}\left(\frac{f_{4}^{10}}{f_{2}^{2} f_{8}^{4}}-4 q \frac{f_{2}^{2} f_{8}^{4}}{f_{4}^{2}}\right) \quad(\bmod 5)
\end{aligned}
$$

Extracting odd powers of $q$ on both sides, we obtain

$$
\begin{aligned}
\sum_{n \geq 0} \bar{t}^{(4)}(8 n+6) q^{n} & \equiv 2 \frac{f_{4}^{6}}{f_{2}^{3}} \quad(\bmod 5) \\
& \equiv 2 \psi^{3}\left(q^{2}\right) \quad(\bmod 5)
\end{aligned}
$$

If we extract even and odd powers of $q$ on both sides of the above equation, we arrive at (5.18) and (5.19), respectively.

\section{Congruences for $\bar{t}^{(7)}(n)$}

In this section, we prove congruences and infinite family of congruences modulo 2 and 4 for $\bar{t}^{(7)}(n)$.

Theorem 6.1 If $n$ is any nonnegative integer, then

$$
\bar{t}^{(7)}(8 n+7) \equiv 0 \quad(\bmod 4) .
$$

Proof Setting $k=7$ in (1.18), we find that

$$
\sum_{n \geq 0} \bar{t}^{(7)}(n) q^{n}=\frac{f_{8}}{f_{1} f_{7}} .
$$

Substituting (2.10) into (6.2) and then extracting the terms involving odd powers of $q$ on both sides of the resulting equation, we obtain

$$
\sum_{n \geq 0} \bar{t}^{(7)}(2 n+1) q^{n}=\frac{f_{2}^{2} f_{4} f_{14}^{2}}{f_{1}^{3} f_{7}^{3}}
$$

In view of (3.10), we can rewrite (6.3) as

$$
\sum_{n \geq 0} \bar{t}^{(7)}(2 n+1) q^{n} \equiv f_{1} f_{4} f_{7} \quad(\bmod 4)
$$

Invoking (2.11) and (6.4), we deduce that

$$
\sum_{n \geq 0} \bar{t}^{(7)}(2 n+1) q^{n} \equiv \frac{f_{2} f_{14} f_{16}^{2} f_{56}^{5}}{f_{8} f_{28}^{3} f_{112}^{2}}-q f_{4}^{2} f_{28}+q^{6} \frac{f_{2} f_{8}^{5} f_{14} f_{112}^{2}}{f_{4}^{2} f_{16}^{2} f_{28} f_{56}} \quad(\bmod 4)
$$

Equating the coefficients of odd powers of $q$ on both sides of the above equation, we find that

$$
\sum_{n \geq 0} \bar{t}^{(7)}(4 n+3) q^{n} \equiv 3 f_{2}^{2} f_{14} \quad(\bmod 4)
$$

which yields the desired result (6.1). 
Theorem 6.2 Let $p \geq 5$ be any prime with $\left(\frac{-14}{p}\right)=-1$ and $1 \leq j \leq p-1$. Then for any nonnegative integers $\alpha$,

$$
\sum_{n \geq 0} \bar{t}^{(7)}\left(8 p^{2 \alpha} n+3 p^{2 \alpha}\right) q^{n} \equiv f_{2} f_{7} \quad(\bmod 2)
$$

and for each $n \geq 0$,

$$
\bar{t}^{(7)}\left(8 p^{2 \alpha+1}(p n+j)+3 p^{2 \alpha+2}\right) \equiv 0 \quad(\bmod 2) .
$$

Proof We prove (6.7) by mathematical induction. In view of (3.9), we can rewrite (6.6) as

$$
\sum_{n \geq 0} \bar{t}^{(7)}(4 n+3) q^{n} \equiv f_{4} f_{14} \quad(\bmod 2)
$$

which yields

$$
\sum_{n \geq 0} \bar{t}^{(7)}(8 n+3) q^{n} \equiv f_{2} f_{7} \quad(\bmod 2)
$$

which is the $\alpha=0$ case of (6.7). Now assume that (6.7) holds for some $\alpha \geq 0$. Substituting (2.13) into (6.7), we deduce that

$$
\begin{aligned}
& \sum_{n \geq 0} \bar{t}^{(7)}\left(8 p^{2 \alpha} n+3 p^{2 \alpha}\right) q^{n} \\
& \equiv\left(\sum_{\substack{k=-\frac{p-1}{2} \\
k \neq( \pm p-1) / 6}}^{\frac{p-1}{2}} q^{3 k^{2}+k} f\left(-q^{3 p^{2}+(6 k+1) p},-q^{3 p^{2}-(6 k+1) p}\right)+q^{\frac{p^{2}-1}{12}} f_{2 p^{2}}\right) \\
& \times\left(\sum_{\substack{k=-\frac{p-1}{2} \\
k \neq( \pm p-1) / 6}}^{\frac{p-1}{2}} q^{7 \times \frac{3 k^{2}+k}{2}} f\left(-q^{7 \times \frac{3 p^{2}+(6 k+1) p}{2}},-q^{7 \times \frac{3 p^{2}-(6 k+1) p}{2}}\right)\right. \\
& \left.+q^{7 \times \frac{p^{2}-1}{24}} f_{7 p^{2}}\right) \quad(\bmod 2) \text {. }
\end{aligned}
$$

For a prime $p \geq 5,-(p-1) / 2 \leq k, m \leq(p-1) / 2$, consider the congruence

$$
2 \times \frac{3 k^{2}+k}{2}+7 \times \frac{3 m^{2}+m}{2} \equiv \frac{9 p^{2}-9}{24}(\bmod p),
$$

that is

$$
(12 k+2)^{2}+14(6 m+1)^{2} \equiv 0(\bmod p) .
$$

Since $\left(\frac{-14}{p}\right)=-1$, the only solution of the above congruence is $k=m=( \pm p-1) / 6$. Therefore, extracting the terms containing $q^{p n+\frac{9 p^{2}-9}{24}}$ from both sides of (6.11), dividing 
throughout by $q^{\frac{9 p^{2}-9}{24}}$ and then replacing $q^{p}$ by $q$, we find that

$$
\sum_{n \geq 0} \bar{t}^{(7)}\left(8 p^{2 \alpha+1} n+3 p^{2 \alpha+2}\right) q^{n} \equiv f_{2 p} f_{7 p} \quad(\bmod 2),
$$

which yields

$$
\sum_{n \geq 0} \bar{t}^{(7)}\left(8 p^{2 \alpha+2} n+3 p^{2 \alpha+2}\right) q^{n} \equiv f_{2} f_{7} \quad(\bmod 2),
$$

which is the (6.7) with $\alpha+1$ for $\alpha$. Comparing the coefficients of $q^{p n+j}$, for $1 \leq j \leq p-1$, from both sides of (6.13), we arrive at (6.8).

\section{Congruences for $\bar{t}^{(8)}(n)$}

In this section, we prove congruences and infinite family of congruences modulo 3,6 , and 12 for $\bar{t}^{(8)}(n)$.

Theorem 7.1 For each nonnegative integer n,

$$
\begin{aligned}
\bar{t}^{(8)}(4 n+3) \equiv 0 & (\bmod 3), \\
\bar{t}^{(8)}(24 n+15) \equiv 0 & (\bmod 12), \\
\bar{t}^{(8)}(24 n+23) \equiv 0 & (\bmod 12) .
\end{aligned}
$$

Proof Setting $k=8$ in (1.18), we obtain

$$
\sum_{n \geq 0} \bar{t}^{(8)}(n) q^{n}=\frac{f_{9}}{f_{8} f_{1}} .
$$

Invoking (2.8) and (7.4), we find that

$$
\sum_{n \geq 0} \bar{t}^{(8)}(n) q^{n}=\frac{f_{12}^{3} f_{18}}{f_{2}^{2} f_{6} f_{8} f_{36}}+q \frac{f_{4}^{2} f_{6} f_{36}}{f_{2}^{3} f_{8} f_{12}} .
$$

Extracting the coefficients of odd powers of $q$ on both sides of the above equation, we obtain

$$
\sum_{n \geq 0} \bar{t}^{(8)}(2 n+1) q^{n}=\frac{f_{2}^{2} f_{3} f_{18}}{f_{1}^{3} f_{4} f_{6}} .
$$

Substituting (2.7) into (7.6) and then extracting the coefficients of odd powers of $q$ on both sides of the resulting equation, we deduce that

$$
\sum_{n \geq 0} \bar{t}^{(8)}(4 n+3) q^{n}=3 \frac{f_{2} f_{6}^{2} f_{9}}{f_{1}^{5}}
$$

from which, we obtain the result (7.1).

In view of (3.10), we have

$$
\frac{f_{2} f_{6}^{2} f_{9}}{f_{1}^{5}} \equiv \frac{f_{6}^{2} f_{9}}{f_{1} f_{2}} \quad(\bmod 4)
$$


Using (7.8), (7.7) can be reduces to

$$
\sum_{n \geq 0} \bar{t}^{(8)}(4 n+3) q^{n} \equiv 3 \frac{f_{6}^{2} f_{9}}{f_{1} f_{2}} \quad(\bmod 12) .
$$

Applying (2.8) in (7.9), we find that

$$
\sum_{n \geq 0} \bar{t}^{(8)}(4 n+3) q^{n} \equiv 3 \frac{f_{6}^{2}}{f_{2}}\left(\frac{f_{18} f_{12}^{3}}{f_{2}^{2} f_{6} f_{36}}+q \frac{f_{4}^{2} f_{6} f_{36}}{f_{2}^{3} f_{12}}\right) \quad(\bmod 12) .
$$

Equating the coefficients of $q^{2 n+1}$, dividing throughout by $q$ and then replacing $q^{2}$ by $q$ in (7.10), we obtain

$$
\sum_{n \geq 0} \bar{t}^{(8)}(8 n+7) q^{n} \equiv 3 \frac{f_{2}^{2} f_{3}^{3} f_{18}}{f_{1}^{4} f_{6}} \quad(\bmod 12)
$$

But, from (3.10)

$$
\frac{f_{2}^{2} f_{3}^{3} f_{18}}{f_{1}^{4} f_{6}} \equiv \frac{f_{3}^{3} f_{18}}{f_{6}} \quad(\bmod 4) .
$$

Invoking (7.11) and (7.12), we find that

$$
\sum_{n \geq 0} \bar{t}^{(8)}(8 n+7) q^{n} \equiv 3 \frac{f_{3}^{3} f_{18}}{f_{6}} \quad(\bmod 12),
$$

which yields the desired results (7.2) and (7.3).

Theorem 7.2 For any prime $p \geq 5$ with $\left(\frac{-6}{p}\right)=-1,1 \leq j \leq p-1, \alpha \geq 0$, we have

$$
\sum_{n \geq 0} \bar{t}^{(8)}\left(24 p^{2 \alpha} n+7 p^{2 \alpha}\right) q^{n} \equiv 3 f_{1} f_{6} \quad(\bmod 6)
$$

and for each $n \geq 0$,

$$
\bar{t}^{(8)}\left(24 p^{2 \alpha+1}(p n+j)+7 p^{2 \alpha+2}\right) \equiv 0 \quad(\bmod 6) .
$$

Proof From (3.9), we have

$$
\frac{f_{3}^{3} f_{18}}{f_{6}} \equiv f_{3} f_{18} \quad(\bmod 2) .
$$

Using (7.16), we can rewrite (7.13) as

$$
\sum_{n \geq 0} \bar{t}^{(8)}(8 n+7) q^{n} \equiv 3 f_{3} f_{18} \quad(\bmod 6),
$$

which yields

$$
\sum_{n \geq 0} \bar{t}^{(8)}(24 n+7) q^{n} \equiv 3 f_{1} f_{6} \quad(\bmod 6) .
$$

For a prime $p \geq 5$ and $-(p-1) / 2 \leq k, m \leq(p-1) / 2$, consider

$$
\frac{3 k^{2}+k}{2}+6 \times \frac{3 m^{2}+m}{2} \equiv \frac{7 p^{2}-7}{24}(\bmod p) \text {, }
$$


which is equivalent to

$$
(6 k+1)^{2}+6(6 m+1)^{2} \equiv 0(\bmod p) .
$$

Since $\left(\frac{-6}{p}\right)=-1$, the only solution of the above congruence is $k=m=( \pm p-1) / 6$. Therefore, using Lemma 2.3, we have

$$
\sum_{n \geq 0} \bar{t}^{(8)}\left(24\left(p^{2} n+\frac{7 p^{2}-7}{24}\right)+7\right) q^{n} \equiv 3 f_{1} f_{6} \quad(\bmod 6) .
$$

Invoking (7.18) and (7.19), we arrive at

$$
\sum_{n \geq 0} \bar{t}^{(8)}\left(24 p^{2} n+7 p^{2}\right) q^{n} \equiv \sum_{n=0}^{\infty} \bar{t}^{(8)}(24 n+7) q^{n} \equiv 3 f_{1} f_{6} \quad(\bmod 6) .
$$

The result (7.14) follows from the above equation and by induction on $\alpha$.

Substituting (2.13) into (7.14), we deduce that

$$
\begin{aligned}
& \sum_{n \geq 0} \bar{t}^{(8)}\left(24 p^{2 \alpha} n+7 p^{2 \alpha}\right) q^{n} \\
& \equiv 3\left(\sum_{\substack{k=-\frac{p-1}{2} \\
k \neq( \pm p-1) / 6}}^{\frac{p-1}{2}} q^{\frac{3 k^{2}+k}{2}} f\left(-q^{\frac{3 p^{2}+(6 k+1) p}{2}},-q^{\frac{3 p^{2}-(6 k+1) p}{2}}\right)+q^{\frac{p^{2}-1}{24}} f_{p^{2}}\right) \\
& \quad \times\left(\sum_{\substack{k=-\frac{p-1}{2} \\
k \neq( \pm p-1) / 6}}^{\frac{p-1}{2}} q^{6 \times \frac{3 k^{2}+k}{2}} f\left(-q^{6 \times \frac{3 p^{2}+(6 k+1) p}{2}},-q^{6 \times \frac{3 p^{2}-(6 k+1) p}{2}}\right)\right. \\
& +q^{\left.6 \times \frac{p^{2}-1}{24} f_{6 p^{2}}\right) \quad(\bmod 6)}
\end{aligned}
$$

which yields

$$
\sum_{n \geq 0} \bar{t}^{(8)}\left(24 p^{2 \alpha+1} n+7 p^{2 \alpha+2}\right) q^{n} \equiv 3 f_{p} f_{6 p} \quad(\bmod 6) .
$$

Equating the coefficients of $q^{p n+j}$ for $j=1,2, \ldots, p-1$ in (7.22), we obtain (7.15).

Acknowledgements The authors would like to thank the referees for helpfull suggestions and comments.

\section{References}

1. Ahmed, Z., Baruah, N.D.: New congruences for Andrews's singular overpartitions. Int. J. Number Theory (2015). https://doi.org/10.1142/S1793042115501018

2. Andrews, G.E.: The Theory of Partitions. Cambridge Univ. Press, Cambridge (1998)

3. Andrews, G.E.: Singular overpartitions. Int. J. Number Theory 5(11), 1523-1533 (2015)

4. Baruah, N.D., Ojah, K.K.: Analogues of Ramanujan's partition identities and congruences arising from his theta functions and modular equations. Ramanujan J. 28, 385-407 (2012)

5. Berndt, B.C.: Ramanujan's Notebooks, Part III. Springer, New York (1991)

6. Bringmann, K., Dousse, J., Lovejoy, J., Mahlburg, K.: Overpartitions with restricted odd differences. Electron. J. Comb. 22(3), 3-17 (2015) 
7. Chen, S.C., Hirschhorn, M.D., Sellers, J.A.: Arithmetic properties of Andrews' singular overpartitions. Int. J. Number Theory 5(11), 1463-1476 (2015)

8. Chern, S., Hao, L.-J.: Congruences for two restricted overpartitions. Proc. Indian Acad. Sci. Math. Sci. (to appear)

9. Corteel, S., Lovejoy, J.: Overpartitions. Trans. Am. Math. Soc. 356, 1623-1635 (2004)

10. Cui, S.P., Gu, N.S.S.: Arithmetic properties of $l$-regular partitions. Adv. Appl. Math. 51, 507-523 (2013)

11. Hirschhorn, M.D., Garvan, F., Borwein, J.: Cubic analogs of the Jacobian cubic theta function $\theta(z, q)$. Can. J. Math. 45, 673-694 (1993)

12. Hirschhorn, M.D., Sellers, J.A.: Elementary proofs of parity results for 5-regular partitions. Bull. Aust. Math. Soc. 81, 58-63 (2010)

13. Mahadeva Naika, M.S., Gireesh, D.S.: Congruences for Andrew's singular overpartitions. J. Number Theory 165, 109-130 (2016)

14. Ramanujan, S.: Some properties of $p(n)$, the number of partitions of $n$. Proc. Camb. Philos. Soc. 19, 207-210 (1919)

15. Ramanujan, S.: Collected Papers. Cambridge Univ. Press, Cambridge (1927)

16. Xia, E.X.W.: New congruences modulo powers of 2 for broken 3-diamond partitions and 7-core partitions. J. Number Theory 141, 119-135 (2014)

17. Xia, E.X.W., Yao, O.X.M.: New Ramanujan-like congruences modulo powers of 2 and 3 for overpartitions. J. Number Theory 133, 1932-1949 (2013)

18. Xia, E.X.W., Yao, O.X.M.: Some modular relations for the Göllnitz-Gordon functions by an even-odd method. J. Math. Anal. Appl. 387, 126-138 (2012) 